\title{
STOCHASTIC INTENSITY FOR MINIMAL REPAIRS IN HETEROGENEOUS POPULATIONS
}

\author{
JI HWAN CHA, * Ewha Womans University \\ MAXIM FINKELSTEIN, ${ }^{* *}$ University of the Free State and Max Planck \\ Institute for Demographic Research
}

\begin{abstract}
In this note we revisit the discussion on minimal repair in heterogeneous populations in Finkelstein (2004). We consider the corresponding stochastic intensities (intensity processes) for items in heterogeneous populations given available information on their operational history, i.e. the failure (repair) times and the time since the last failure (repair). Based on the improved definitions, the setup of Finkelstein (2004) is modified and the main results are corrected in accordance with the updating procedure for the conditional frailty distribution.
\end{abstract}

Keywords: Heterogeneous population; minimal repair; frailty; intensity process; updating procedure

2010 Mathematics Subject Classification: Primary 60K10

Secondary 90B25

\section{Introduction}

Consider a system with an absolutely continuous time-to-failure cumulative distribution function (CDF) $F(t)$ and a failure rate $\lambda(t)$, which starts operating at $t=0$. Assume that the repair action is performed instantaneously upon failure. The repair is usually qualified as perfect if the CDF of the repaired object is $F(t)$ (as good as new) and as minimal at time $x$ if its $\mathrm{CDF}$ is

$$
F(t \mid x) \equiv 1-\frac{1-F(t+x)}{1-F(x)}
$$

(as bad as old). Thus, the minimal repair restores our system to the state (statistically, i.e. defined by the $\mathrm{CDF}$ ) it had prior to the failure. In practice, the minimal repair can be performed using the following 'operations'.

(A) The failed system is replaced by a statistically identical system (with the same CDF) that was operating for the same time but did not fail.

(B) The system consists of a large number of elementary components and, therefore, the replacement of only the failed component by an as-good-as-new component does not essentially change the system's failure rate. The minimal repair is considered as a reasonable approximation in this case.

Received 7 January 2011; revision received 7 April 2011.

* Postal address: Department of Statistics, Ewha Womans University, Seoul, 120-750, Korea.

Email address: jhcha@ewha.ac.kr

** Postal address: Department of Mathematical Statistics, University of the Free State, 339 Bloemfontein 9300, South Africa. Email address: finkelm@ufs.ac.za 
Sometimes, upon failure, we can observe additional information about the state of an object (e.g. the structure of a system). This can allow us to define a more general type of repair, which is usually called the information-based (or physical) minimal repair. The information-based minimal repair brings our object back to the state (to be defined by the relevant information) it had just prior to the failure (see Bergman (1985), Arjas and Noros (1989), Natvig (1990), Finkelstein (1992), (2004), Boland and El-Neweihi (1998), and Aven and Jensen (1999, pp. 82 87)).

It is very challenging to generalize the notion of minimal repair to items from heterogeneous populations. An attempt was performed in Finkelstein (2004), where some useful considerations and approaches were discussed. Three scenarios for different types of minimal repair were considered, and the corresponding intensity processes were obtained and compared. However, the considered updating procedure for the frailty distribution in Finkelstein (2004) used only a part of the available information about the failures and survival of items, and, therefore, the corresponding mathematical model had to be modified and the main results had to be corrected accordingly. Thus, the main objective of our note is, using the developed updating procedure for frailty distributions, to modify and correct these results and to describe the modeling in a more consistent way.

The structure of this note is as follows. In Section 2 we revisit the paper by Finkelstein (2004) and define the intensity process for heterogeneous populations in a more consistent way. Based on the improved definitions, in Sections 3 and 4, the corresponding models and results of Finkelstein (2004) are modified and corrected. Furthermore, a useful interpretation of the obtained results is discussed. Finally, concluding remarks are given in Section 5.

\section{The setting and interpretation}

Let failures of repairable items be repaired instantaneously. Then the process of repairs can be described by a stochastic point process. A convenient mathematical description of these processes uses the concept of stochastic intensity (the intensity process) $\lambda_{t}, t \geq 0$ (see Aven and Jensen (2000)). As discussed, e.g. in Finkelstein (2008, pp. 71-72), the stochastic intensity of an orderly point process $N(t), t \geq 0$, is defined as the limit

$$
\lambda_{t}=\lim _{\Delta t \rightarrow 0} \frac{\operatorname{Pr}\left[N(t, t+\Delta t)=1 \mid H_{t}\right]}{\Delta t}=\lim _{\Delta t \rightarrow 0} \frac{\mathrm{E}\left[N(t, t+\Delta t) \mid H_{t}\right]}{\Delta t},
$$

where $H_{t}=\{N(s): 0 \leq s<t\}$ is an internal filtration (history) of the point process in $[0, t)$, i.e. the set of all point events in $[0, t)$.

A classical example of $\lambda_{t}$ is the intensity process generated by the renewal process (perfect repairs)

$$
\lambda_{t}=\sum_{n=0}^{\infty} \lambda\left(t-T_{n}\right) \mathbf{1}\left(T_{n} \leq t<T_{n+1}\right), \quad T_{0}=0,
$$

where $T_{1}<T_{2}<T_{3}<\cdots$ are the random failure times. Another standard example is the 'deterministic stochastic intensity' $\lambda_{t}=\lambda(t)$ which defines the nonhomogeneous Poisson process (NHPP) of repairs with intensity $\lambda(t)$. It is well known that, in accordance with definition (1), this example can also be interpreted as the process of minimal repairs.

As in Finkelstein (2004), (2008, pp. 133-135), we formally describe heterogeneous populations in the following way. Let $T \geq 0$ be a lifetime random variable (RV) with CDF $F(t)$ $(\bar{F}(t) \equiv 1-F(t))$. Assume that $F(t)$ is indexed by an $\mathrm{RV} Z$, i.e.

$$
\operatorname{Pr}(T \leq t \mid Z=z) \equiv \operatorname{Pr}(T \leq t \mid z) \equiv F(t, z)
$$


and that the probability density function (PDF) $f(t, z)$ exists. Then the corresponding failure rate $\lambda(t, z)$ is $f(t, z) / \bar{F}(t, z)$. Let $Z$ be a nonnegative RV with support in $[a, b], 0 \leq a<$ $b \leq \infty$, and PDF $\pi(z)$. A meaningful interpretation defines the unobserved $Z$ as the frailty in the heterogeneous population. The above setting leads naturally to considering mixtures of distributions, which are useful for describing heterogeneity:

$$
F_{\mathrm{m}}(t)=\int_{a}^{b} F(t, z) \pi(z) \mathrm{d} z .
$$

The mixture failure rate in accordance with the definition is

$$
\lambda_{\mathrm{m}}(t)=\frac{\int_{a}^{b} f(t, z) \pi(z) \mathrm{d} z}{\int_{a}^{b} \bar{F}(t, z) \pi(z) \mathrm{d} z}=\int_{a}^{b} \lambda(t, z) \pi(z \mid t) \mathrm{d} z,
$$

where $\pi(z \mid t)$ is the conditional PDF (on condition that $T>t$ ) defined by

$$
\pi(z \mid t) \equiv \pi(z \mid T>t)=\pi(z) \frac{\bar{F}(t, z)}{\int_{a}^{b} \bar{F}(t, z) \pi(z) \mathrm{d} z} .
$$

Similarly to Finkelstein (2004), who proceeded using examples, we now define two types (scenarios) of minimal repair for heterogeneous populations, but in a more general context. The first type of minimal repair does not employ any additional information and, therefore, the failed item is replaced by a statistically (in distribution) identical item. As the failure time distribution in this case is just the mixture (3), the stochastic intensity for the corresponding process of minimal repairs of this type is obviously equal to the mixture failure rate, i.e.

$$
\lambda_{t}=\lambda_{\mathrm{m}}(t), \quad t \geq 0 .
$$

The second type of minimal repair (already information based) restores an item to a statistically identical item with the same value of (unobserved) frailty $Z$. It can be realized in practice by performing 'operation' (B), resulting in the 'classical' minimal repair in which only a small part of a large system is replaced. It is natural to suggest that the state of an item is also defined by the corresponding realization of the frailty parameter (i.e. if $Z=z$ before the failure, it should be $z$ after the failure). Thus, (1) is modified to

$$
F(t, z \mid x) \equiv 1-\frac{1-F(t+x, z)}{1-F(x, z)} .
$$

Our attention in this note mainly centers on this type of minimal repair, as it is the most 'interesting' from both a practical and theoretical point of view.

Remark 1. In Finkelstein (2004), a third type of minimal repair was also defined that differed from the second type by only the assumption that the frailty $Z$ is 'observed'. The following reasoning shows that the stochastic intensity in this case is the same as for the second type of minimal repair and, therefore, there is no need to consider the third type of minimal repair (from a modeling point of view).

Let us return to definition (2) and modify it with respect to the 'heterogeneous' case when the orderly point process is indexed by the frailty parameter $Z$ (see the corresponding interpretation 
after the formal definition (6) below). Observe that the stochastic intensity $\lambda_{t}$ (unconditional with respect to the frailty $Z$ ) can now be specified as

$$
\begin{aligned}
\lambda_{t} & =\lim _{\Delta t \rightarrow 0} \frac{\mathrm{E}\left[\operatorname{Pr}\left[N(t, t+\Delta t)=1 \mid H_{t}, Z\right]\right]}{\Delta t} \\
& =\mathrm{E}\left[\lim _{\Delta t \rightarrow 0} \frac{\operatorname{Pr}\left[N(t, t+\Delta t)=1 \mid H_{t}, Z\right]}{\Delta t}\right] \\
& =\mathrm{E}\left[\lambda_{t, Z}\right],
\end{aligned}
$$

where the expectation is with respect to the conditional distribution $Z \mid H_{t}$ and

$$
\lambda_{t, Z} \equiv \lim _{\Delta t \rightarrow 0} \frac{\operatorname{Pr}\left[N(t, t+\Delta t)=1 \mid H_{t}, Z\right]}{\Delta t} .
$$

Then $\lambda_{t, z}(Z=z)$ in (6) can be interpreted as the conditional (with respect to $Z$ ) stochastic intensity of the orderly point process, indexed by the frailty $Z$.

We now specify our point process (i.e. the second type of minimal repair process). As before, let $Z$ be the frailty of an item randomly selected at time $t=0$ from our heterogeneous population. Upon each failure, perform the second type of minimal repair. Note that in this case if $Z=z$ at time $t=0$ then the corresponding realization is $\lambda_{t, z}=\lambda(t, z)$ for all $t \geq 0$ (whether or not the randomly selected frailty $Z$ is observable). Therefore, for the second type of minimal repair, $\lambda_{t, Z}$ in (6) is now given by

$$
\lambda_{t, Z}=\lambda(t, Z), \quad t \geq 0,
$$

and, in accordance with (5), the corresponding stochastic intensity $\lambda_{t}$ is the expectation of $\lambda(t, Z)$ with respect to the distribution of $Z \mid H_{t}$. This operation means that although the value of $Z$ is chosen at $t=0$ and is fixed, its distribution is updated with time as information about the failures and survival times emerges (see the detailed procedure in the next section).

\section{Updating the frailty}

We see that stochastic modeling for the second type of minimal repair is dramatically different from that for the first type, as information about the operational history (failure times and survival times) in fact updates the conditional frailty distribution $Z \mid H_{t}$.

In accordance with our considerations, it is clear that the stochastic intensity $\lambda_{t}=\mathrm{E}\left[\lambda_{t}, Z\right]$ defined in (5) for $t \in\left[0, t_{1}\right)$, where $t_{1}$ is the realization of the failure time $T_{1}$, is just the mixture failure rate (4), i.e. $\lambda_{\mathrm{m}}^{1}(t)=\lambda_{\mathrm{m}}(t)$, as the information at hand is just the initial distribution $\pi(z)$ (and the fact that the item has survived in $[0, t)$ ).

Now consider the next interval $\left[t_{1}, t_{2}\right)$. Given the additional information (in addition to the initial distribution $\pi(z)$ ) that an item has failed at $t=t_{1}$, the PDF of the frailty $Z=z$ (we repair the item to the state defined by the same value of the frailty) is

$$
\pi_{02}(z) \equiv \frac{\lambda\left(t_{1}, z\right) \exp \left\{-\int_{0}^{t_{1}} \lambda(s, z) \mathrm{d} s\right\} \pi(z)}{\int_{a}^{b} \lambda\left(t_{1}, z\right) \exp \left\{-\int_{0}^{t_{1}} \lambda(s, z) \mathrm{d} s\right\} \pi(z) \mathrm{d} z} .
$$

Thus, the 'initial frailty distribution' (at the start of the second cycle) just after the minimal repair is given by (7). Furthermore, the 'remaining survival function' at time $t=t_{1}$ is given by 
$\left[\bar{F}\left(t_{1}+u, z\right) / \bar{F}\left(t_{1}, z\right)\right]$. Then, the conditional frailty distribution $Z \mid H_{t}$ in $\left[t_{1}, t_{2}\right)$ is

$$
\frac{\left[\bar{F}(t, z) / \bar{F}\left(t_{1}, z\right)\right] \pi_{02}(z)}{\int_{a}^{b}\left[\bar{F}(t, z) / \bar{F}\left(t_{1}, z\right)\right] \pi_{02}(z) \mathrm{d} z}=\frac{\lambda\left(t_{1}, z\right) \exp \left\{-\int_{0}^{t} \lambda(s, z) \mathrm{d} s\right\} \pi(z)}{\int_{a}^{b} \lambda\left(t_{1}, z\right) \exp \left\{-\int_{0}^{t} \lambda(s, z) \mathrm{d} s\right\} \pi(z) \mathrm{d} z},
$$

and the corresponding stochastic intensity is, in accordance with (5),

$$
\lambda_{\mathrm{m}}^{2}(t)=\int_{a}^{b} \lambda(t, z) \frac{\lambda\left(t_{1}, z\right) \exp \left\{-\int_{0}^{t} \lambda(s, z) \mathrm{d} s\right\} \pi(z)}{\int_{a}^{b} \lambda\left(t_{1}, z\right) \exp \left\{-\int_{0}^{t} \lambda(s, z) \mathrm{d} s\right\} \pi(z) \mathrm{d} z} \mathrm{~d} z \quad \text { in }\left[t_{1}, t_{2}\right) .
$$

Using another useful (Bayesian) interpretation, we can reason that the item fails at time $t_{1}$ and, after repair, survives in $\left[t_{1}, t\right]$. Thus, the corresponding probability (conditional probability given $Z=z$ at $t=0$ ) is

$$
\begin{gathered}
\lambda\left(t_{1}, z\right) \exp \left\{-\int_{0}^{t_{1}} \lambda(s, z) \mathrm{d} s\right\} \exp \left\{-\int_{t_{1}}^{t} \lambda(s, z) \mathrm{d} s\right\} \mathrm{d} t_{1} \\
=\lambda\left(t_{1}, z\right) \exp \left\{-\int_{0}^{t} \lambda(s, z) \mathrm{d} s\right\} \mathrm{d} t_{1} .
\end{gathered}
$$

Given this information, the conditional frailty distribution $Z \mid H_{t}$ should be updated as

$$
\frac{\lambda\left(t_{1}, z\right) \exp \left\{-\int_{0}^{t} \lambda(s, z) \mathrm{d} s\right\} \pi(z)}{\int_{a}^{b} \lambda\left(t_{1}, z\right) \exp \left\{-\int_{0}^{t} \lambda(s, z) \mathrm{d} s\right\} \pi(z) \mathrm{d} z},
$$

which yields (8). Note that the updating procedure in Finkelstein (2004, Equations (7) and (8)) was dependent only on survival in $\left[t_{1}, t\right]$ and not on the full operational history in $[0, t)$. This remark also refers to the following steps of updating.

Consider now the intensity process in $\left[t_{2}, t_{3}\right)$. For $\left[t_{2}, t_{3}\right)$, as we know that the item failed at times $t_{1}$ and $t_{2}$, and, after minimal repairs, survived to $t-t_{2}$, the corresponding probability (conditional probability given $Z=z$ at $t=0$, divided by $\mathrm{d} t_{1} \mathrm{~d} t_{2}$ ) is

$$
\begin{gathered}
\lambda\left(t_{1}, z\right) \exp \left\{-\int_{0}^{t_{1}} \lambda(s, z) \mathrm{d} s\right\} \lambda\left(t_{2}, z\right) \exp \left\{-\int_{t_{1}}^{t_{2}} \lambda(s, z) \mathrm{d} s\right\} \exp \left\{-\int_{t_{2}}^{t} \lambda(s, z) \mathrm{d} s\right\} \\
=\lambda\left(t_{1}, z\right) \lambda\left(t_{2}, z\right) \exp \left\{-\int_{0}^{t} \lambda(s, z) \mathrm{d} s\right\} .
\end{gathered}
$$

Given this information, the conditional frailty distribution $Z \mid H_{t}$ should be updated as

$$
\frac{\lambda\left(t_{1}, z\right) \lambda\left(t_{2}, z\right) \exp \left\{-\int_{0}^{t} \lambda(s, z) \mathrm{d} s\right\} \pi(z)}{\int_{a}^{b} \lambda\left(t_{1}, z\right) \lambda\left(t_{2}, z\right) \exp \left\{-\int_{0}^{t} \lambda(s, z) \mathrm{d} s\right\} \pi(z) \mathrm{d} z} .
$$

Thus, in $\left[t_{2}, t_{3}\right)$, as before,

$$
\lambda_{\mathrm{m}}^{3}(t)=\int_{a}^{b} \lambda(t, z) \frac{\lambda\left(t_{1}, z\right) \lambda\left(t_{2}, z\right) \exp \left\{-\int_{0}^{t} \lambda(s, z) \mathrm{d} s\right\} \pi(z)}{\int_{a}^{b} \lambda\left(t_{1}, z\right) \lambda\left(t_{2}, z\right) \exp \left\{-\int_{0}^{t} \lambda(s, z) \mathrm{d} s\right\} \pi(z) \mathrm{d} z} \mathrm{~d} z \quad \text { in }\left[t_{2}, t_{3}\right) .
$$

More generally, for $t \in\left[t_{n-1}, t_{n}\right)$, the conditional frailty distribution $Z \mid H_{t}$ is defined by

$$
\pi^{n}\left(z \mid t_{1}, \ldots, t_{n-1}\right) \equiv \frac{\lambda\left(t_{1}, z\right) \cdots \lambda\left(t_{n-1}, z\right) \exp \left\{-\int_{0}^{t} \lambda(s, z) \mathrm{d} s\right\} \pi(z)}{\int_{a}^{b} \lambda\left(t_{1}, z\right) \cdots \lambda\left(t_{n-1}, z\right) \exp \left\{-\int_{0}^{t} \lambda(s, z) \mathrm{d} s\right\} \pi(z) \mathrm{d} z}
$$


and, therefore,

$$
\lambda_{\mathrm{m}}^{n}(t)=\int_{a}^{b} \lambda(t, z) \pi^{n}\left(z \mid t_{1}, \ldots, t_{n-1}\right) \mathrm{d} z \quad \text { in }\left[t_{n-1}, t_{n}\right) .
$$

Based on (9) and (10), the corresponding stochastic intensity can now be defined as

$$
\lambda_{t}=\sum_{n=1}^{\infty} \lambda_{\mathrm{m}}^{n}(t) \mathbf{1}\left(T_{n-1} \leq t<T_{n}\right), \quad T_{0} \equiv 0 .
$$

Therefore, $\pi\left(z \mid t-t_{n-1}\right)$ in Equation (10) of Finkelstein (2004) should be corrected as $\pi^{n}\left(z \mid t_{1}, \ldots, t_{n-1}\right)$.

\section{Comparing stochastic intensities}

The following result presents a useful ordering of stochastic intensities for minimal repairs of the first and second types.

Theorem 1. Let the values of $\lambda(t, z)$ be ordered with respect to $z$, i.e. for all $z_{1}, z_{2} \in[a, b]$ and $t \geq 0$,

$$
\lambda\left(t, z_{1}\right)<\lambda\left(t, z_{2}\right) \text { if } z_{1}<z_{2} .
$$

Then

$$
\lambda_{\mathrm{m}}(t) \leq \lambda_{t}, \quad t \geq 0,
$$

where $\lambda_{t}$ is the stochastic intensity for the second type of minimal repair in (11).

Proof. Note that if $X \leq_{\mathrm{st}} Y$ and $g(\cdot)$ is any increasing function, then $g(X) \leq_{\mathrm{st}} g(Y)$ and, accordingly, $\mathrm{E}[g(X)] \leq \mathrm{E}[g(Y)]$. Observe that both $\lambda_{\mathrm{m}}(t)$ and $\lambda_{t}$ are expectations of $\lambda(t, Z)$ with respect to the mixing distributions

$$
\pi(z \mid t)=\pi(z) \frac{\bar{F}(t, z)}{\int_{a}^{b} \bar{F}(t, z) \pi(z) \mathrm{d} z}
$$

and

$$
\pi^{n}\left(z \mid t_{1}, \ldots, t_{n-1}\right) \equiv \frac{\lambda\left(t_{1}, z\right) \cdots \lambda\left(t_{n-1}, z\right) \exp \left\{-\int_{0}^{t} \lambda(s, z) \mathrm{d} s\right\} \pi(z)}{\int_{a}^{b} \lambda\left(t_{1}, z\right) \cdots \lambda\left(t_{n-1}, z\right) \exp \left\{-\int_{0}^{t} \lambda(s, z) \mathrm{d} s\right\} \pi(z) \mathrm{d} z},
$$

respectively. Then it is sufficient to show that

$$
\Pi(v \mid t) \geq \Pi^{n}\left(v \mid t_{1}, \ldots, t_{n-1}\right)
$$

for all $n \geq 1$ and $0<t_{1}<\cdots<t_{n-1}<t$, where $\Pi(v \mid t)$ and $\Pi^{n}\left(v \mid t_{1}, \ldots, t_{n-1}\right)$ are the corresponding CDFs. Observe that

$$
\begin{aligned}
\pi^{n}\left(z \mid t_{1}, \ldots, t_{n-1}\right) & \equiv \frac{\lambda\left(t_{1}, z\right) \cdots \lambda\left(t_{n-1}, z\right) \bar{F}(t, z) \pi(z)}{\int_{a}^{b} \lambda\left(t_{1}, z\right) \cdots \lambda\left(t_{n-1}, z\right) \bar{F}(t, z) \pi(z) \mathrm{d} z} \\
& =\frac{\lambda\left(t_{1}, z\right) \cdots \lambda\left(t_{n-1}, z\right) \pi(z \mid t)}{\int_{a}^{b} \lambda\left(t_{1}, z\right) \cdots \lambda\left(t_{n-1}, z\right) \pi(z \mid t) \mathrm{d} z} .
\end{aligned}
$$


It is clear that there exist $a \leq z^{*}(a, v) \leq v$ and $v \leq z^{*}(v, b) \leq b$ such that

$$
\int_{a}^{v} \lambda\left(t_{1}, z\right) \cdots \lambda\left(t_{n-1}, z\right) \pi(z \mid t) \mathrm{d} z=\lambda\left(t_{1}, z^{*}(a, v)\right) \cdots \lambda\left(t_{n-1}, z^{*}(a, v)\right) \int_{a}^{v} \pi(z \mid t) \mathrm{d} z
$$

and

$$
\int_{v}^{b} \lambda\left(t_{1}, z\right) \cdots \lambda\left(t_{n-1}, z\right) \pi(z \mid t) \mathrm{d} z=\lambda\left(t_{1}, z^{*}(v, b)\right) \cdots \lambda\left(t_{n-1}, z^{*}(v, b)\right) \int_{v}^{b} \pi(z \mid t) \mathrm{d} z .
$$

Thus,

$$
\begin{aligned}
\Pi^{n}\left(v \mid t_{1}, \ldots, t_{n-1}\right)= & \left(\lambda\left(t_{1}, z^{*}(a, v)\right) \cdots \lambda\left(t_{n-1}, z^{*}(a, v)\right) \int_{a}^{v} \pi(z \mid t) \mathrm{d} z\right) \\
& \times\left(\lambda\left(t_{1}, z^{*}(a, v)\right) \cdots \lambda\left(t_{n-1}, z^{*}(a, v)\right) \int_{a}^{v} \pi(z \mid t) \mathrm{d} z\right. \\
& \left.\quad+\lambda\left(t_{1}, z^{*}(v, b)\right) \cdots \lambda\left(t_{n-1}, z^{*}(v, b)\right) \int_{v}^{b} \pi(z \mid t) \mathrm{d} z\right)^{-1} \\
\leq & \int_{a}^{v} \pi(z \mid t) \mathrm{d} z \\
= & \Pi(v \mid t),
\end{aligned}
$$

where the inequality follows from the fact that, since $\lambda\left(t_{1}, z\right) \cdots \lambda\left(t_{n-1}, z\right)$ is an increasing function of $z$,

$$
\lambda\left(t_{1}, z^{*}(a, v)\right) \cdots \lambda\left(t_{n-1}, z^{*}(a, v)\right) \leq \lambda\left(t_{1}, z^{*}(v, b)\right) \cdots \lambda\left(t_{n-1}, z^{*}(v, b)\right) ;
$$

therefore, inequality (12) is justified.

The following example is a correction of Example 2 of Finkelstein (2004).

Example 1. Suppose that $F(t, z)$ is an exponential distribution with parameter $\lambda(t, z)=z \lambda$, and let $\pi(z)$ be an exponential PDF in $[0, \infty)$ with parameter $\theta$. Then, from $(4), \lambda_{\mathrm{m}}(t)=$ $\lambda /(\lambda t+\theta)$. Observe that

$$
\begin{aligned}
\pi^{n}\left(z \mid t_{1}, \ldots, t_{n-1}\right) & \equiv \frac{\lambda\left(t_{1}, z\right) \cdots \lambda\left(t_{n-1}, z\right) \exp \left\{-\int_{0}^{t} \lambda(s, z) \mathrm{d} s\right\} \pi(z)}{\int_{a}^{b} \lambda\left(t_{1}, z\right) \cdots \lambda\left(t_{n-1}, z\right) \exp \left\{-\int_{0}^{t} \lambda(s, z) \mathrm{d} s\right\} \pi(z) \mathrm{d} z} \\
& =\frac{(z \lambda)^{n-1} \exp \{-z \lambda t\} \theta \exp \{-\theta z\}}{\int_{0}^{\infty}(z \lambda)^{n-1} \exp \{-z \lambda t\} \theta \exp \{-\theta z\} \mathrm{d} z},
\end{aligned}
$$

and, from (9) and (10),

$$
\lambda_{\mathrm{m}}^{n}(t)=\frac{\int_{0}^{\infty}(z \lambda)^{n} \exp \{-(\lambda t+\theta) z\} \mathrm{d} z}{\int_{0}^{\infty}(z \lambda)^{n-1} \exp \{-(\lambda t+\theta) z\} \mathrm{d} z}=n \frac{\lambda}{\lambda t+\theta} .
$$

Finally, from (11),

$$
\lambda_{t}=\sum_{n=1}^{\infty} n \frac{\lambda}{\lambda t+\theta} \mathbf{1}\left(T_{n-1} \leq t<T_{n}\right), \quad T_{0} \equiv 0 .
$$

Thus, $\lambda_{\mathrm{m}}(t) \leq \lambda_{t}, t \geq 0$, holds. 
Denote by $H_{\mathrm{m}}(t)$ and $H_{\lambda}(t)$ the mean numbers of repairs (failures) in [0,t) that correspond to the minimal repair processes of type 1 and type 2, respectively. The following result obviously follows from Theorem 1 .

Corollary 1. Under the assumptions of Theorem 1,

$$
H_{\mathrm{m}}(t) \leq H_{\lambda}(t)
$$

In addition to the theoretical comparison of stochastic intensities for two types of minimal repair, the following remark suggests another practical application of this ordering.

Remark 2. Assume that the population of items under consideration is heterogeneous and is stochastically described by (3) and (4). The user, who is performing the minimal repair (by replacing only the failed part of large systems, which is described as 'operation' (B) in the introduction), however, does not know (or does not take into account) the heterogeneity structure of the population and considers it to be homogeneous with failure distribution $F_{\mathrm{m}}(t)$ and failure rate $\lambda_{\mathrm{m}}(t)$. In accordance with our results, $\lambda_{\mathrm{m}}(t) \leq \lambda_{t}$ and $H_{\mathrm{m}}(t) \leq H_{\lambda}(t)$, which specifically means that the mean number of minimal repairs in reality is larger than that for the homogeneous model. This underestimation can often be 'dangerous' in reliability practice (e.g. when assessing the number of spare parts required).

\section{Concluding remarks}

In this note the stochastic intensities for two types of minimal repair in heterogeneous populations have been considered. The first type is, in fact, the statistical minimal repair that does not employ any additional information, whereas the second type is the information-based minimal repair, which restores an item to a statistically identical item with the same value of (unobserved) frailty $Z$. The setup of Finkelstein (2004) is modified and the main results are corrected in accordance with the developed updating procedure for the conditional frailty distribution.

It has been shown that the stochastic intensity for the second type of minimal repair is larger (with probability 1) than the deterministic stochastic intensity that corresponds to the first type of minimal repair. This implies that the mean number of minimal repairs for the heterogeneous setting is larger than that for the corresponding homogeneous model, which can be useful in reliability applications.

\section{Acknowledgements}

The authors would like to thank the anonymous referee for very helpful, detailed suggestions on the presentation of this paper. The work of the first author was supported by the Priority Research Centers Program through the National Research Foundation of Korea, funded by the Ministry of Education, Science and Technology (grant number 2009-0093827). The work of the second author was supported by the National Research Foundation of South Africa (grant number FA2006040700002).

\section{References}

ArJas, E. AND Norros, I. (1989). Change of life distribution via hazard transformation: an inequality with application to minimal repair. Math. Operat. Res. 14, 355-361.

Aven, T. And Jensen, U. (1999). Stochastic Models in Reliability (Appl. Math. 41). Springer, New York.

Aven, T. And Jensen, U. (2000). A general minimal repair model. J. Appl. Prob. 37, 187-197. 
Bergman, B. (1985). On reliability theory and its applications. Scand. J. Statist. 12, 1-41.

Boland, P. J. ANd El-Neweini, E. (1998). Statistical and information based (physical) minimal repair for $k$ out of $n$ systems. J. Appl. Prob. 35, 731-740.

Finkelstein, M. S. (1992). Some notes on two types of minimal repair. Adv. Appl. Prob. 24, 226-228.

Finkelstein, M. S. (2004). Minimal repair in heterogeneous populations. J. Appl. Prob. 41, 281-286.

Finkelstein, M. (2008). Failure Rate Modelling for Reliability and Risk. Springer, London.

Natvig, B. (1990). On information-based minimal repair and the reduction in remaining system lifetime due to the failure of a specific module. J. Appl. Prob. 27, 365-375. 